\title{
A note on uniform approximation of functions having a double pole
}

\author{
Ionela Moale and Veronika Pillwein
}

\begin{abstract}
We consider the classical problem of finding the best uniform approximation by polynomials of $1 /(x-a)^{2}$, where $a>1$ is given, on the interval [-1,1]. First, using symbolic computation tools we derive the explicit expressions of the polynomials of best approximation of low degrees and then give a parametric solution of the problem in terms of elliptic functions. Symbolic computation is invoked then once more to derive a recurrence relation for the coefficients of the polynomials of best uniform approximation based on a Pell-type equation satisfied by the solutions.
\end{abstract}

\section{Introduction}

The aim of this paper is to show how symbolic computation tools can be integrated into the study of classical problems in uniform approximation theory. To exemplify this, we consider the problem of finding the polynomial of best uniform approximation of the function $1 /(x-a)^{2}$, where $a>1$ is given, on the interval $[-1,1]$; more precisely, we are looking for $p_{n}^{*} \in \Pi_{n}$, where $\Pi_{n}:=\left\{p_{n}: p_{n}(x)=\sum_{i=0}^{n} c_{i} x^{i}, c_{i} \in \mathbb{R}\right\}$, such that

$$
\begin{aligned}
\left\|\frac{1}{(x-a)^{2}}-p_{n}^{*}\right\| & :=\max _{x \in[-1,1]}\left|\frac{1}{(x-a)^{2}}-p_{n}^{*}(x)\right| \\
& =\min _{p_{n} \in \Pi_{n}} \max _{x \in[-1,1]}\left|\frac{1}{(x-a)^{2}}-p_{n}(x)\right| .
\end{aligned}
$$

We call $p_{n}^{*}$ the polynomial of best approximation and

$$
y_{n}(x):=\frac{1}{(x-a)^{2}}-p_{n}^{*}(x) \text { and } L_{n}:=\left\|\frac{1}{(x-a)^{2}}-p_{n}^{*}(x)\right\|
$$

the error function of best approximation and the minimum deviation, respectively.

An asymptotic expression for the minimum deviation is well-known, for example [5, p. 122],

$$
L_{n} \sim \frac{n}{\left(a^{2}-1\right)^{(n+1) / 2}} \frac{1}{\left(a+\sqrt{a^{2}-1}\right)^{n}},
$$

however explicit expressions for the error function of best approximation and the minimum deviation do not seem to appear in the classical textbooks on approximation theory; we mention here $[\mathbf{1}, \mathbf{2}, \mathbf{5}]$ and also the more recent $[\mathbf{6}]$. The reason behind this omission is most probably the fact that not all of the parameters involved in the description of the solution, which can be represented in terms of elliptic functions, can be given explicitly. As we will also see here, a pair of parameters is defined as the solution of a system of equations rather involved, but one which can be easily solved numerically in any of the available computer algebra systems.

Received 19 November 2012; revised 5 December 2013.

2010 Mathematics Subject Classification 13P10, 33E05, 41A10, 65D20, 68W30 (primary).

The authors were partially supported by the Austrian Science Fund (FWF) under grant P22748-N18. The first named author was supported by the strategic program 'Innovatives OÖ 2010plus' by the Upper Austrian Government. 
The case of a function having a simple pole, namely $1 /(x-a)$, where $a>1$ is given, is significantly simpler, explicit expressions for the polynomial of best approximation as well as for the minimum deviation in terms of elementary functions being well-known; see, for example, [5, p. 120]. These polynomials were recently used in $[\mathbf{1 5}, \mathbf{1 6}]$ to provide simple convergence analysis for the algebraic multilevel methods, a three-term recurrence relation for the polynomials being derived for this analysis. Naturally it would be interesting to know whether the polynomials of best approximation corresponding to the problem (1) also satisfy such a recurrence relation, however we will not dwell on this here, but rather study recurrence relations for their coefficients.

The paper is organized as follows: in $\S 2$ we give the explicit solution of the problem (1) in terms of elliptic functions as well as explicit expressions for the polynomials of lower degrees. Section 3 is devoted to the proof of the explicit solution and some additional remarks. In the concluding $\S 4$ we comment on how to derive a recurrence relation for the coefficients of the polynomials of best approximation.

\section{Main results}

By the Chebyshev alternation theorem (see, for example, [1, p. 55]), the error function of best approximation $y_{n}(x)$ must have at least $n+2$ alternation points in the interval $[-1,1]$. It is easy to show that the derivative of a function of the form $1 /(x-a)^{2}+p_{n}(x), p_{n} \in \Pi_{n}$, has at most $n$ real zeros. Hence $y_{n}^{\prime}(x)$ has precisely $n$ distinct real zeros $\xi_{1}, \ldots, \xi_{n} \in(-1,1)$ and two complex conjugate zeros $z_{1}, z_{2}$, and thus the alternation points of $y_{n}(x)$ are $-1<\xi_{1}<\ldots<\xi_{n}<1$. In addition, $y_{n}(x)$ decreases from $+\infty$ to $-\infty$ on the interval $(a, \infty)$.

With this clear view of how the graph of the error function of best approximation looks, we determined with the help of Mathematica the explicit expressions of $p_{n}^{*}(x)$ and $L_{n}$ for $n=1$ and $n=2$ and arbitrary $a>1$; see also Figure 2. More precisely,

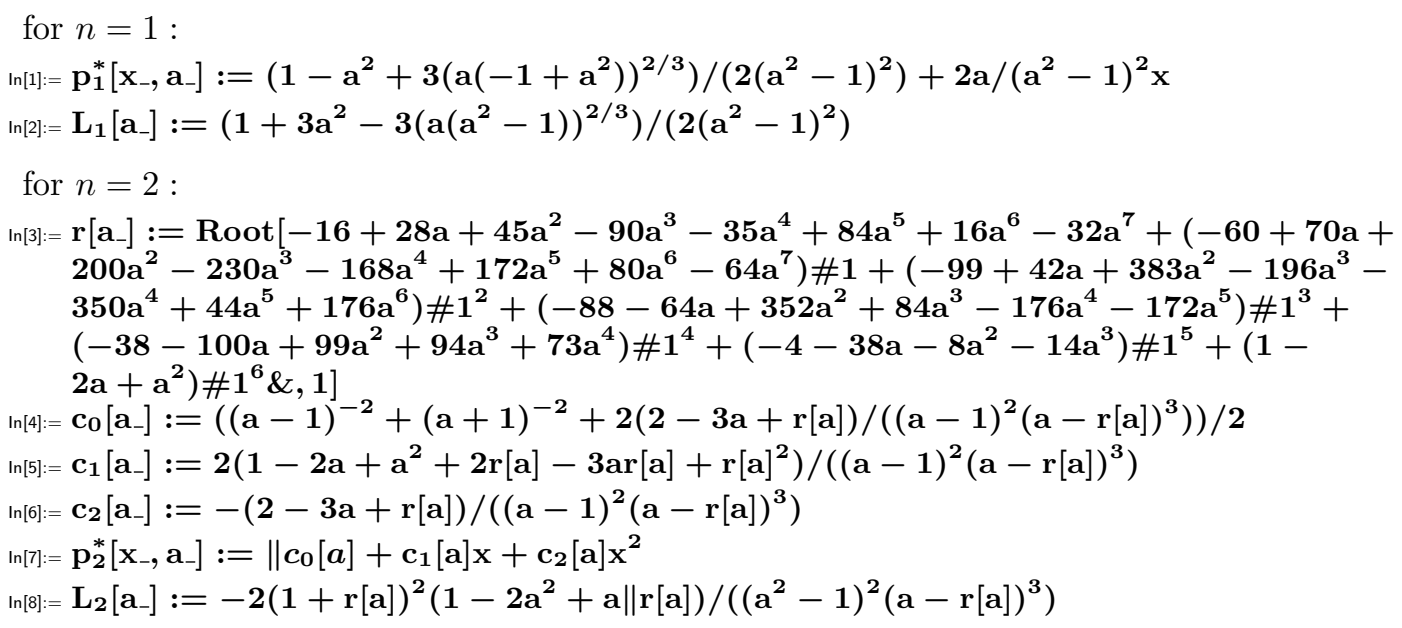

For the computation of these explicit formulas Gröbner bases $[\mathbf{8}, \mathbf{1 0}, \mathbf{2 0}]$ were used, a wellestablished tool in symbolic computation for solving systems of polynomial equations. Gröbner bases computations may be computationally very expensive, depending exponentially on the number of variables in the input and also badly on the polynomial degrees. Finding the linear polynomial of best approximation is an easy task that can be solved on any computer algebra system. However, starting from degree two, the computations become very involved and we used Singular [11] via a Mathematica interface [12].

In order to determine the polynomial of best approximation, we use a generic ansatz for the polynomial $p_{n}^{*}(x)=\sum_{k=0}^{n} c_{k} x^{k}$. Using the fact that the $n+2$ alternation points are $-1, \xi_{1}, \ldots, \xi_{n}, 1$ (sorted in increasing order) and that the interior alternation points $\xi_{j}$ 

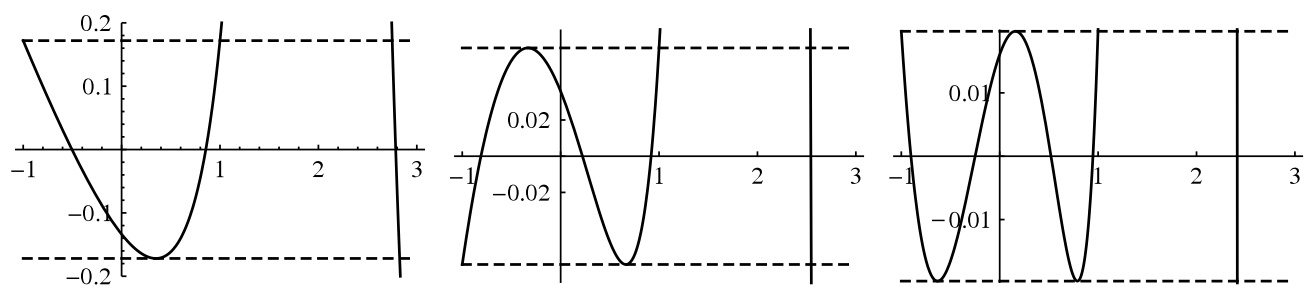

FiguRE 1. Graphs of $y_{1}(x), y_{2}(x)$ and $y_{3}(x)$ for $a=2$.

are extreme points, so that their first derivative vanishes, we obtain a system of $2 n+1$ equations in the $2 n+1$ unknowns $c_{0}, \ldots, c_{n}, \xi_{1}, \ldots, \xi_{n}$ :

$$
\begin{gathered}
y_{n}(-1)=(-1)^{n+1} y_{n}(1) \quad \text { and } \quad y_{n}\left(\xi_{j}\right)=(-1)^{n+j} y_{n}(1), \quad 1 \leqslant j \leqslant n \\
y_{n}^{\prime}\left(\xi_{j}\right)=0, \quad 1 \leqslant j \leqslant n .
\end{gathered}
$$

Even though ultimately we only need to determine the coefficients $c_{k}$, currently we do not see how to set up a system without using the alternation points. Bringing everything to a common denominator and equating the numerators of these equations to zero yields the polynomial system. The coefficients $c_{k}$ appear only linearly and the $\xi_{k}$ appear in monomials up to degree $n+2$. This increase in numbers of variables and degrees explains why we reach a limit in our computations quickly. For $n=2$ it is still possible to obtain the coefficients comparably fast for symbolic $a$. Also for degree three it is still possible to obtain the coefficients in reasonable time (less than $10 \mathrm{~min}$ ) for specific choices of $a$. Note that these choices are not floating point numbers and the result is still exact and can be evaluated to arbitrary precision. Starting from degree four we could not find closed-form solutions, not even for specific choices of $a$.

For $n=3$ and $a=2$ the coefficients of the polynomial of best approximation are algebraic numbers that are approximately given by

$$
c_{0}=0.2339547125, \quad c_{1}=0.2062172304, \quad c_{2}=0.3018857394, \quad c_{3}=0.2382272140 .
$$

The minimal polynomial for, for example, $c_{3}$ is given by

$$
\begin{aligned}
\chi(\alpha)= & 215892499727278669824 \alpha^{11}-5459947971497905618944 \alpha^{10} \\
& +1699378437698250434795520 \alpha^{9}+4985576127353627620088832 \alpha^{8} \\
& -1487598201477303010010019 \alpha^{7}+49652231802046011126432 \alpha^{6} \\
& -128259012822418301760 \alpha^{5}-39565695530318777856 \alpha^{4} \\
& +1028117822899995648 \alpha^{3}-23136252868952064 \alpha^{2} \\
& +277724396519424 \alpha-1099511627776 .
\end{aligned}
$$

A Gröbner basis is a basis for the polynomial ideal spanned by the given set of polynomials that is unique once a monomial ordering on the variables has been fixed. The basis is normalized to be monic and it is auto-reduced. If the monomial ordering is lexicographic, then a Gröbner basis has the elimination property, that is, if we are computing in the polynomial ring $\mathbb{K}\left[x_{1}, \ldots, x_{n}\right]$ (for some field $\mathbb{K}$ ) with a lexicographic ordering such that $x_{1}<\ldots<x_{n}$, then $($ with $k<n$ )

$$
I \cap \mathbb{K}\left[x_{1}, \ldots, x_{k}\right]=\left\langle G \cap \mathbb{K}\left[x_{1}, \ldots, x_{k}\right]\right\rangle,
$$

where $I$ denotes the ideal spanned by the input, $G$ its Gröbner basis and $\langle S\rangle$ denotes the polynomial ideal spanned by the set $S$. This property allows us to use Gröbner bases for solving polynomial systems of equations. 
In our computations in the first step we eliminate the variables $c_{k}$ from the given system. Certainly it would be desirable to eliminate the $\xi_{k}$ and solve the remaining equations for the coefficients, however this is computationally too expensive. In the second step, we determine the solutions $\xi_{k}$ of the remaining equations. From this solution set we pick the solution satisfying $-1<\xi_{1}<\ldots<\xi_{n}<1$. Plugging into the original system gives the coefficients $c_{k}$ we are after.

The following theorem gives the parametric solution to the problem (1) for arbitrary $n \in \mathbb{N}$. As usual $K:=K(k)$ is the complete integral of the first kind of modulus $k, 0<k<1$, $K^{\prime}:=K^{\prime}(k):=K\left(k^{\prime}\right)$ where $k^{\prime}:=\sqrt{1-k^{2}}, \operatorname{sn}(u):=\operatorname{sn}(u ; k)$ is the Jacobi elliptic function and $\Theta(u):=\Theta(u ; k), \mathrm{H}(u):=\mathrm{H}(u ; k), \mathrm{H}_{1}(u):=\mathrm{H}_{1}(u ; k)$ and $\Theta_{1}(u):=\Theta_{1}(u ; k)$ are the four Jacobi theta functions; see, for example, $[\mathbf{2}, \mathbf{6}]$.

Theorem 2.1. Let $k, 0<k<1$, and $\rho, 0<\rho<K / n$, be defined by the system

$$
\left\{\begin{array}{l}
\left(n(a-1)+\frac{2(a-\alpha)(a-\beta)-\left(a^{2}-1\right)(2 a-\alpha-\beta)}{\sqrt{\left(a^{2}-1\right)(a-\alpha)(a-\beta)}}\right) \frac{\operatorname{cn} \rho \operatorname{dn} \rho}{\operatorname{sn} \rho} \\
-2 n \frac{\Theta^{\prime}(\rho)}{\Theta(\rho)}-4 \frac{\Theta^{\prime}\left(\hat{\rho}+i K^{\prime}\right)}{\Theta\left(\hat{\rho}+i K^{\prime}\right)}-\frac{2 \pi i}{K}=0 \\
\operatorname{sn}\left(\hat{\rho}+i K^{\prime}\right)-\sqrt{\frac{a+1}{a-1}} \operatorname{sn} \rho=0,
\end{array}\right.
$$

where

$$
\alpha=\frac{1+k^{2} \operatorname{sn}^{2} \rho}{1-k^{2} \operatorname{sn}^{2} \rho}, \quad \beta=\frac{1+\operatorname{sn}^{2} \rho}{1-\operatorname{sn}^{2} \rho} \quad \text { and } \quad \hat{\rho}=\frac{K}{2}-\frac{n}{2} \rho,
$$

and let

$$
x=\frac{\operatorname{sn}^{2} u+\operatorname{sn}^{2} \rho}{\operatorname{sn}^{2} u-\operatorname{sn}^{2} \rho} .
$$

Then the solution of the approximation problem is

$$
\begin{aligned}
& y_{n}(x)=(-1)^{n-1} \frac{L_{n}}{2}\left\{\left[\frac{\mathrm{H}(\rho+u)}{\mathrm{H}(\rho-u)}\right]^{n}\left[\frac{\Theta(\hat{\rho}+u)}{\Theta(\hat{\rho}-u)}\right]^{2}+\left[\frac{\mathrm{H}(\rho-u)}{\mathrm{H}(\rho+u)}\right]^{n}\left[\frac{\Theta(\hat{\rho}-u)}{\Theta(\hat{\rho}+u)}\right]^{2}\right\}, \\
& L_{n}=\frac{-e^{-(\pi / 2)\left(K^{\prime} / K\right)}}{2} \frac{\left[\Theta^{\prime}\left(-i K^{\prime}\right)\right]^{2}}{k^{\prime 2}} \frac{[\Theta(\rho-\hat{\rho})]^{n+4}}{[\Theta(\rho+\hat{\rho})]^{n-4}} \frac{\Theta^{6}(0)}{\Theta_{1}^{2}(0) \mathrm{H}_{1}^{2}(0) \Theta^{4}(\rho) \mathrm{H}^{4}(\rho) \mathrm{H}^{4}(2 \hat{\rho})} .
\end{aligned}
$$

The proof of the theorem given in the next section is based on the functional equation (9) satisfied by the error function of best approximation $y_{n}(x)$, recalling Pell's equation from number theory. This equation is the key tool in deriving a recurrence relation for the coefficients of $p_{n}^{*}(x)$, see $\S 4$.

Pell-type equations are satisfied by many polynomials appearing in approximation theory, such as Chebyshev polynomials, Zolotarev polynomials, and Achieser polynomials; see, for example, $[\mathbf{1}]$. We refer to the survey paper $[\mathbf{1 8}]$ for further details on this topic. Therefore, the approach we used here to derive a recurrence relation for the coefficients of the polynomials of best approximation to $1 /(x-a)^{2}, a>1$, can be carried over also to the above-mentioned cases.

\section{Proof of Theorem 2.1}

The key tool in obtaining the solution of the approximation problem (1) we are considering here is a Pell-type equation satisfied by the error function of best approximation $y_{n}(x)$. 
To write down this equation, let $a<\alpha<\beta$ be such that $y_{n}(\alpha)=-y_{n}(\beta)=L_{n}$. Based on the explanation from the beginning of the previous section and denoting $p_{n}^{*}(x)=$ $c_{n} x^{n}+c_{n-1} x^{n-1}+\ldots+c_{0}$, we have

$$
\begin{aligned}
{\left[y_{n}(x)\right]^{2}-L_{n}^{2} } & =c_{n}^{2} \frac{\left(x-\xi_{1}\right)^{2} \ldots\left(x-\xi_{n}\right)^{2}\left(x^{2}-1\right)(x-\alpha)(x-\beta)}{(x-a)^{4}} \\
y_{n}^{\prime}(x) & =-n c_{n} \frac{\left(x-\xi_{1}\right) \ldots\left(x-\xi_{n}\right)\left(x-z_{1}\right)\left(x-z_{2}\right)}{(x-a)^{3}} .
\end{aligned}
$$

Combining the two relations, we conclude that $y_{n}(x)$ satisfies the following Pell-type equation:

$$
\frac{\left[y_{n}^{\prime}(x)\right]^{2}}{\left[y_{n}(x)\right]^{2}-L_{n}^{2}}=n^{2} \frac{\left(x^{2}-\gamma_{1} x+\gamma_{2}\right)^{2}}{(x-a)^{2}} \frac{1}{\left(x^{2}-1\right)(x-\alpha)(x-\beta)}
$$

where $\gamma_{1}:=z_{1}+z_{2}, \gamma_{2}:=z_{1} z_{2}$. The formulas of $\gamma_{1}$ and $\gamma_{2}$ can be easily obtained using the explicit form of $y_{n}(x)=1 /(x-a)^{2}-\left(c_{n} x^{n}+\ldots c_{0}\right)$ and of $y_{n}^{\prime}(x)$ derived from this one, in combination with equations (8), namely

$$
\begin{aligned}
& \gamma_{1}=2 a-\frac{2 a(a-\alpha)(a-\beta)+\left(a^{2}-1\right)(2 a-(\alpha+\beta))}{n \sqrt{\left(a^{2}-1\right)(a-\alpha)(a-\beta)}} \\
& \gamma_{2}=a^{2}-\frac{2(a-\alpha)(a-\beta)+a\left(a^{2}-1\right)(2 a-(\alpha+\beta))}{n \sqrt{\left(a^{2}-1\right)(a-\alpha)(a-\beta)}} .
\end{aligned}
$$

Equation (9) implies that

$$
\log \frac{y_{n}(x)+\sqrt{y_{n}^{2}(x)-L_{n}^{2}}}{ \pm L_{n}}=\int_{-1}^{x} \frac{n\left(x^{2}-\gamma_{1} x+\gamma_{2}\right)}{(x-a) \sqrt{\left(x^{2}-1\right)(x-\alpha)(x-\beta)}} d x
$$

where on the right-hand side we have an elliptic integral, which we solve by making a suitable substitution. For this, let $x(u)$ be the conformal mapping from the rectangle with vertices at the points $u=0, K, K+i K^{\prime}, i K^{\prime}$, with $K$ and $K^{\prime}$ of modulus $k, 0<k<1$, onto the lower half-plane with the following normalization:

$$
x(0)=-1, \quad x\left(i K^{\prime}\right)=1, \quad x\left(K+i K^{\prime}\right)=\alpha, \quad x(K)=\beta .
$$

The conformal mapping is given by

$$
x(u)=\frac{\mathrm{sn}^{2} u+\mathrm{sn}^{2} \rho}{\operatorname{sn}^{2} u-\operatorname{sn}^{2} \rho},
$$

where $k, 0<k<1$, and $\rho, 0<\rho<K$, are defined by

$$
k=\sqrt{\frac{\alpha-1}{\alpha+1} \frac{\beta+1}{\beta-1}} \quad \text { and } \quad \operatorname{sn}^{2} \rho=\frac{\beta-1}{\beta+1} .
$$

The expression (12) was found as $x=g \circ f$, where $f$ is the conformal mapping from the rectangle with vertices at the points $u=0, K, K+i K^{\prime}, i K^{\prime}$, onto the upper half-plane with the normalization $f(0)=0, f(K)=1, f\left(K+i K^{\prime}\right)=1 / k^{2}, f\left(i K^{\prime}\right)=\infty$, and $g$ is the conformal mapping of the upper half-plane onto the lower half-plane with the normalization $g(0)=-1, g(1)=\beta, g(\infty)=1, g\left(1 / k^{2}\right)=\alpha$. We have $f(u)=\operatorname{sn}^{2} u$, by writing first the expression of the inverse map with the help of the Schwarz-Christoffel formula, and $g(u)=$ $((\beta+1) u+(\beta-1)) /((\beta+1) u-(\beta-1))$ obtained as a linear fractional transform satisfying 
the first three normalizing conditions and then obtaining $k$ from the last one. The substitution we use in the computation of the elliptic integral in (11) is then

$$
x=\frac{z^{2}+\lambda^{2}}{z^{2}-\lambda^{2}} \quad \text { with } \lambda^{2}=\frac{\beta-1}{\beta+1}, \quad k^{2}=\frac{\alpha-1}{\alpha+1} \frac{\beta+1}{\beta-1},
$$

which implies

$$
\alpha=\frac{1+k^{2} \operatorname{sn}^{2} \rho}{1-k^{2} \operatorname{sn}^{2} \rho}, \quad \beta=\frac{1+\operatorname{sn}^{2} \rho}{1-\operatorname{sn}^{2} \rho} .
$$

Therefore,

$$
\begin{aligned}
& \int_{-1}^{x} \frac{x^{2}-\gamma_{1} x+\gamma_{2}}{(x-a) \sqrt{\left(x^{2}-1\right)(x-\alpha)(x-\beta)}} d x \\
& \quad=\frac{1}{a-1} \frac{\sqrt{\left(1-\lambda^{2}\right)\left(1-k^{2} \lambda^{2}\right)}}{\lambda} \int_{0}^{z} \frac{\left(1-\gamma_{1}+\gamma_{2}\right) z^{4}+2\left(1-\gamma_{2}\right) \lambda^{2} z^{2}+\left(1+\gamma_{1}+\gamma_{2}\right) \lambda^{4}}{\left(z^{2}-\lambda^{2}\right)\left(z^{2}-\frac{a+1}{a-1} \lambda^{2}\right) \sqrt{\left(1-z^{2}\right)\left(1-k^{2} z^{2}\right)}} d z,
\end{aligned}
$$

and, after partial fraction decomposition, we arrive at

$$
\begin{aligned}
\int_{-1}^{x} & \frac{x^{2}-\gamma_{1} x+\gamma_{2}}{(x-a) \sqrt{\left(x^{2}-1\right)(x-\alpha)(x-\beta)}} d x \\
= & \frac{1-\gamma_{1}+\gamma_{2}}{a-1} \frac{\sqrt{\left(1-\lambda^{2}\right)\left(1-k^{2} \lambda^{2}\right)}}{\lambda} \int_{0}^{z} \frac{d z}{\sqrt{\left(1-z^{2}\right)\left(1-k^{2} z^{2}\right)}} \\
& -2 \lambda \sqrt{\left(1-\lambda^{2}\right)\left(1-k^{2} \lambda^{2}\right)} \int_{0}^{z} \frac{d z}{\left(z^{2}-\lambda^{2}\right) \sqrt{\left(1-z^{2}\right)\left(1-k^{2} z^{2}\right)}} \\
& +\frac{2\left(a^{2}-a \gamma_{1}+\gamma_{2}\right)}{(a-1)^{2}} \lambda \sqrt{\left(1-\lambda^{2}\right)\left(1-k^{2} \lambda^{2}\right)} \int_{0}^{z} \frac{d z}{\left(z^{2}-\frac{a+1}{a-1} \lambda^{2}\right) \sqrt{\left(1-z^{2}\right)\left(1-k^{2} z^{2}\right)}}
\end{aligned}
$$

For the second and third integral in the last equality above, let $\rho$ and $\tilde{\rho}$ be defined by $\operatorname{sn} \rho=\lambda$ and sn $\tilde{\rho}=\sqrt{((a+1) /(a-1))} \lambda$, respectively. We note here that the two conditions imply that

$$
a=\frac{\mathrm{sn}^{2} \tilde{\rho}+\mathrm{sn}^{2} \rho}{\mathrm{sn}^{2} \tilde{\rho}-\mathrm{sn}^{2} \rho}
$$

hence $\tilde{\rho}$ is the pre-image of $a$ through the conformal mapping (12) and thus it is of the form $\tilde{\rho}=\hat{\rho}+i K^{\prime}, \hat{\rho} \in(0, K)$. With the above notation for $\rho$ and using [21, p. 523], it can be easily shown that

$$
\int_{0}^{z} \frac{d z}{\left(z^{2}-\lambda^{2}\right) \sqrt{\left(1-z^{2}\right)\left(1-k^{2} z^{2}\right)}}=\frac{1}{\operatorname{sn} \rho \operatorname{cn} \rho \operatorname{dn} \rho}\left[\frac{1}{2} \log \frac{\mathrm{H}(\rho-u)}{\mathrm{H}(\rho+u)}+\frac{\Theta^{\prime}(\rho)}{\Theta(\rho)} u\right],
$$

with a similar expression holding for the third integral. Therefore, using the fact that

$$
\frac{n\left(a^{2}-a \gamma_{1}+\gamma_{2}\right)}{(a-1)^{2}} \frac{\operatorname{sn} \rho \operatorname{cn} \rho \operatorname{dn} \rho}{\operatorname{sn} \tilde{\rho} \operatorname{cn} \tilde{\rho} \operatorname{dn} \tilde{\rho}}=-2,
$$

we arrive at

$$
\begin{array}{rl}
\int_{-1}^{x} & n \frac{x^{2}-\gamma_{1} x+\gamma_{2}}{(x-a) \sqrt{\left(x^{2}-1\right)(x-\alpha)(x-\beta)}} d x \\
\quad=\left[\frac{n\left(1-\gamma_{1}+\gamma_{2}\right)}{a-1} \frac{\operatorname{cn} \rho \operatorname{dn} \rho}{\operatorname{sn} \rho}-2 n \frac{\Theta^{\prime}(\rho)}{\Theta(\rho)}-4 \frac{\Theta^{\prime}(\tilde{\rho})}{\Theta(\tilde{\rho})}\right] u+n \log \frac{\mathrm{H}(\rho+u)}{\mathrm{H}(\rho-u)}+2 \log \frac{\mathrm{H}(\tilde{\rho}+u)}{\mathrm{H}(\tilde{\rho}-u)} .
\end{array}
$$


Taking into account also that $\tilde{\rho}=\hat{\rho}+i K^{\prime}$ we have thus by (11) and the reduction formulas for Jacobi theta functions:

$$
\begin{aligned}
\log \frac{y_{n}(x)+\sqrt{y_{n}^{2}(x)-L_{n}^{2}}}{ \pm L_{n}}= & {\left[\frac{n\left(1-\gamma_{1}+\gamma_{2}\right)}{a-1} \frac{\operatorname{cn} \rho \operatorname{dn} \rho}{\operatorname{sn} \rho}-2 n \frac{\Theta^{\prime}(\rho)}{\Theta(\rho)}-4 \frac{\Theta^{\prime}\left(\hat{\rho}+i K^{\prime}\right)}{\Theta\left(\hat{\rho}+i K^{\prime}\right)}-\frac{2 \pi i}{K}\right] u } \\
& +\log \left[\frac{\mathrm{H}(\rho+u)}{\mathrm{H}(\rho-u)}\right]^{n}+\log \left[\frac{\Theta(\hat{\rho}+u)}{\Theta(\hat{\rho}-u)}\right]^{2}
\end{aligned}
$$

From the condition that $y_{n}(x)$ has $n+2$ alternation points in $[-1,1]$ we must have

$$
\frac{n\left(1-\gamma_{1}+\gamma_{2}\right)}{a-1} \frac{\operatorname{cn} \rho \operatorname{dn} \rho}{\operatorname{sn} \rho}-2 n \frac{\Theta^{\prime}(\rho)}{\Theta(\rho)}-4 \frac{\Theta^{\prime}\left(\hat{\rho}+i K^{\prime}\right)}{\Theta\left(\hat{\rho}+i K^{\prime}\right)}-\frac{2 \pi i}{K}=\left(n \frac{\rho}{K}+2 \frac{\hat{\rho}}{K}-1\right) \frac{\pi}{K^{\prime}}
$$

and from the condition that $y_{n}(x)$ strictly decreases from $L_{n}$ to $-L_{n}$ in $[\alpha, \beta]$ that

$$
n \frac{\rho}{K}+2 \frac{\hat{\rho}}{K}-1=0
$$

Combining these two we conclude from (15) that

$$
y_{n}(x)=(-1)^{n-1} \frac{L_{n}}{2}\left\{\left[\frac{\mathrm{H}(\rho+u)}{\mathrm{H}(\rho-u)}\right]^{n}\left[\frac{\Theta(\hat{\rho}+u)}{\Theta(\hat{\rho}-u)}\right]^{2}+\left[\frac{\mathrm{H}(\rho-u)}{\mathrm{H}(\rho+u)}\right]^{n}\left[\frac{\Theta(\hat{\rho}-u)}{\Theta(\hat{\rho}+u)}\right]^{2}\right\}
$$

with $\rho$ and $k$ defined by (3) and $\alpha, \beta$ and $\hat{\rho}$ by (4).

The value of $L_{n}$ follows now from the condition that $\lim _{x \rightarrow a}(x-a)^{2} y_{n}(x)=1$. By (12) and (14) we get that $x-a$ is equal to

$$
g(u):=\frac{2 \mathrm{sn}^{2} \rho}{\operatorname{sn}^{2} \rho-\operatorname{sn}^{2}\left(\hat{\rho}+i K^{\prime}\right)} \frac{\operatorname{sn}^{2} u-\operatorname{sn}^{2}\left(\hat{\rho}+i K^{\prime}\right)}{\operatorname{sn}^{2} u-\operatorname{sn}^{2} \rho},
$$

and, therefore, with $f(u)=y_{n}(x(u))$,

$$
L_{n}=\frac{1}{\lim _{u \rightarrow \hat{\rho}+i K^{\prime}} f(u) g(u)^{2}}
$$

which is easily shown to be equal to

$$
L_{n}=\frac{(-1)^{n-1}}{8} \frac{\mathrm{H}^{n}\left(\rho-\hat{\rho}-i K^{\prime}\right)}{\mathrm{H}^{n}\left(\rho+\hat{\rho}+i K^{\prime}\right)} \frac{\left[\Theta^{\prime}\left(-i K^{\prime}\right)\right]^{2}\left(\mathrm{sn}^{2} \rho-\mathrm{sn}^{2}\left(\hat{\rho}+i K^{\prime}\right)\right)^{4}}{\Theta^{2}\left(2 \hat{\rho}+i K^{\prime}\right) \operatorname{sn}^{4} \rho\left[\operatorname{sn}\left(\hat{\rho}+i K^{\prime}\right) \operatorname{cn}\left(\hat{\rho}+i K^{\prime}\right) \operatorname{dn}\left(\hat{\rho}+i K^{\prime}\right)\right]^{2}}
$$

and, after some transformations, to (7).

The proof of the theorem is complete up to the fact that the system (3) has a unique solution which satisfies $0<k<1$ and, since $\rho, \hat{\rho} \in(0, K)$, that $0<\rho<K / n$. We do not consider this matter here, but rather give the numerical solutions of the system for $a=2$ and $n=1,2, \ldots, 8$, which were found with the help of the command FindRoot in Mathematica:

\begin{tabular}{ccc}
\hline$n$ & $k$ & $\rho$ \\
\hline 1 & 0.988912134768707 & 0.846885238283369 \\
2 & 0.998506984080587 & 0.793161228023789 \\
3 & 0.999760160023856 & 0.764276888897998 \\
4 & 0.999957225505635 & 0.745744327757575 \\
5 & 0.999991825727615 & 0.732764470377829 \\
6 & 0.999998359426700 & 0.723151786732571 \\
7 & 0.999999658473260 & 0.715743316327916 \\
8 & 0.999999926858805 & 0.709858166486558 \\
\hline
\end{tabular}


We note that we have chosen to prove the theorem by computing the elliptic integral on the right-hand side of (11), rather than introducing Riemann surfaces and considering rational functions on them, in the hope for availability of algorithms for dealing with such integrals. Even though many integrals over special functions nowadays can be dealt with by symbolic computation $[\mathbf{3}, \mathbf{7}]$, these particular ones are still out of scope. We consider this paper also as a challenge to derive new methods that extend to this class of integrals.

We conclude this section with some remarks concerning the best uniform approximation on the interval $[-1,1]$ of the function $1 /(x-a)^{2}+B /(x-a)$, where $a>1$ and $B \in \mathbb{R}$, by polynomials of degree $n \in \mathbb{N}$.

In the case when $B=0$, the expression of the error function of best approximation was given in Theorem 2.1 in terms of elliptic functions. As we will briefly explain in what follows, with a different choice of the parameters $\rho$ and $k$, this expression describes the solution also when $B$ is in some small neighborhood of 0 . More precisely, a function defined by (6), with $L_{n}$ and $\hat{\rho}$ given by (7) and (4), respectively, and with $k \in(0,1)$ and $\rho \in(0, K / n)$ arbitrary, is a rational function of the form $\tilde{y}_{n}(x)=1 /(x-a)^{2}+B /(x-a)+p_{n}(x)$, where $p_{n} \in \Pi_{n}$ and

$$
\begin{gathered}
B=\frac{e^{(\pi i / 4 K)\left(2 K+4 \hat{\rho}+3 i K^{\prime}\right)}}{8\left(1-k^{2}\right)} \frac{\left[\Theta^{\prime}\left(-i K^{\prime}\right)\right]^{2} \Theta^{6}(0) \Theta^{2}(\rho-\hat{\rho}) \Theta^{2}(\rho+\hat{\rho})}{\mathrm{H}^{\prime}(0) \Theta^{2}(\rho) \mathrm{H}^{2}(\rho) \Theta^{2}(\hat{\rho}) \Theta_{1}^{2}(\hat{\rho}) \mathrm{H}^{2}(\hat{\rho}) \mathrm{H}_{1}^{2}(\hat{\rho})}\left[4 \Theta^{\prime}\left(2 \hat{\rho}+i K^{\prime}\right)\right. \\
\left.+\left((n+2) \frac{\mathrm{H}^{\prime}\left(\rho-\hat{\rho}-i K^{\prime}\right)}{\mathrm{H}\left(\rho-\hat{\rho}-i K^{\prime}\right)}+(n-2) \frac{\mathrm{H}^{\prime}\left(\rho+\hat{\rho}+i K^{\prime}\right)}{\mathrm{H}\left(\rho+\hat{\rho}+i K^{\prime}\right)}\right) \Theta\left(2 \hat{\rho}+i K^{\prime}\right)\right] .
\end{gathered}
$$

It is easy to show that indeed also in this general case $y_{n}(x)$ is a rational function with a single pole $x=a$ of order two, while as we already stated, the definition of $L_{n}$ is such that the coefficient of $1 /(x-a)^{2}$ is equal to 1 ; see (17) above. The expression (18) was then found as $B=\lim _{x \rightarrow a}\left(y_{n}(x)(x-a)^{2}-1\right) /(x-a)$, making use of (6) and (16). Now when $u$ runs in the segment from 0 to $i K^{\prime}$, then $|\mathrm{H}(\rho+u) / \mathrm{H}(\rho-u)|=1$ and $|\Theta(\hat{\rho}+u) / \Theta(\hat{\rho}-u)|=1$, hence $\left|y_{n}(x)\right| \leqslant L_{n}$ on $[-1,1]$. In addition, by a simple application of the argument principle, $y_{n}(x)$ alternates at least $n+2$ times between $\pm L_{n}$ on $[-1,1]$. Hence the Chebyshev alternation theorem can be applied, and thus for those values of $B$ in some neighborhood of 0 for which the system in the unknowns $k$ and $\rho$ formed by (18) and the second equation in (3) has a solution, we obtain the best approximation to $1 /(x-a)^{2}+B /(x-a)$. That is, plugging in the solution values for $k$ and $\rho$, then $y_{n}(x)$ defined by (6), with $L_{n}$ given by (7) and $\hat{\rho}$ by (4), is the corresponding error function of best approximation.

On the other hand, in the case when $B=\left(2 a+n \sqrt{a^{2}-1}\right) /\left(a^{2}-1\right)$, the expression of the error function of best approximation $y_{n}(x)$ of $1 /(x-a)^{2}+B /(x-a)$ by polynomials of degree $n$ can be given in terms of elementary functions; namely with $x=(1 / 2)(z+1 / z),|z|=1$, then

$$
y_{n}(x)=\frac{L_{n}}{2}\left\{z^{n}\left(\frac{\lambda-z}{1-\lambda z}\right)^{2}+z^{-n}\left(\frac{1-\lambda z}{\lambda-z}\right)^{2}\right\}, \quad L_{n}=\frac{8 \lambda^{n+4}}{\left(1-\lambda^{2}\right)^{4}}
$$

where $\lambda:=a-\sqrt{a^{2}-1}$. This follows immediately from the Chebyshev alternation theorem, again using the argument principle.

\section{Recurrence relation for the polynomials of best approximation}

With $k$ and $\rho$ defined by the system (3) from Theorem 2.1, we have $\alpha$ and $\beta$ defined by (4), $\gamma_{1}$ and $\gamma_{2}$ defined by (10) and the Pell-type equation (9) for $y_{n}(x)$. By differentiating relation (9) we obtain the following linear differential equation for $y_{n}(x)$ with polynomial coefficients

$$
2 f_{0}(x) f_{1}(x) y_{n}^{\prime \prime}(x)+\left(f_{0}^{\prime}(x) f_{1}(x)-f_{0}(x) f_{1}^{\prime}(x)\right) y_{n}^{\prime}(x)-2 f_{1}^{2}(x) y_{n}(x)=0,
$$


where

$$
f_{0}(x)=\left(x^{2}-1\right)(x-a)^{2}(x-\alpha)(x-\beta) \quad \text { and } \quad f_{1}(x)=n^{2}\left(x^{2}-\gamma_{1} x+\gamma_{2}\right)^{2} .
$$

It is well known that this type of differential equation can be turned into a recurrence relation for the coefficients in the series expansion of $y_{n}(x)$ around $x_{0}=0$. Also the given function $1 /(x-a)^{2}$ satisfies a linear differential equation with polynomial coefficients. Hence, the difference $p_{n}^{*}(x)=1 /(x-a)^{2}-y_{n}(x)$ satisfies such a differential equation and a recurrence for its coefficients in the monomial expansion can be computed. This transfer from differential to recurrence equation can be done entirely automatically using symbolic computation. The framework for this are holonomic functions, that is, functions (discrete and/or continuous) satisfying systems of linear difference/differential relations with polynomial coefficients. Algorithms for executing the transfer between differential equation for the function and recurrence relation for the coefficients or for executing closure properties, for example, given recurrences for two sequences return the recurrence for the termwise sum of these sequences, have been implemented in different computer algebra systems $[\mathbf{9}, \mathbf{1 3}]$. Here we use Koutschan's Mathematica implementation [14] 'HolonomicFunctions'`.

The algorithms implemented in HolonomicFunctions deal with difference/differential equations in operator form over some appropriate algebra. For the underlying computations again Gröbner bases are involved, this time also in non-commutative rings. We use this tool to derive a recurrence relation for the coefficients $b_{k}$ of the power series expansion $y_{n}(x)=\sum_{k \geqslant 0} b_{k} x^{k}$. After loading the package in the first step we translate the given differential equation (19) into operator notation:

$\ln [9]:=\operatorname{ann} Y=$ ToOrePolynomial $[$ ode, $y[x]]$;

Then in the second step we pass from the differential equation of $y_{n}$ to the recurrence relation for the coefficients $b_{k}$ using the 'DFiniteDE2RE' command (differential equation to recurrence equation). The output is again in operator notation, but we can use the 'ApplyOreOperator' command to write it in traditional form:

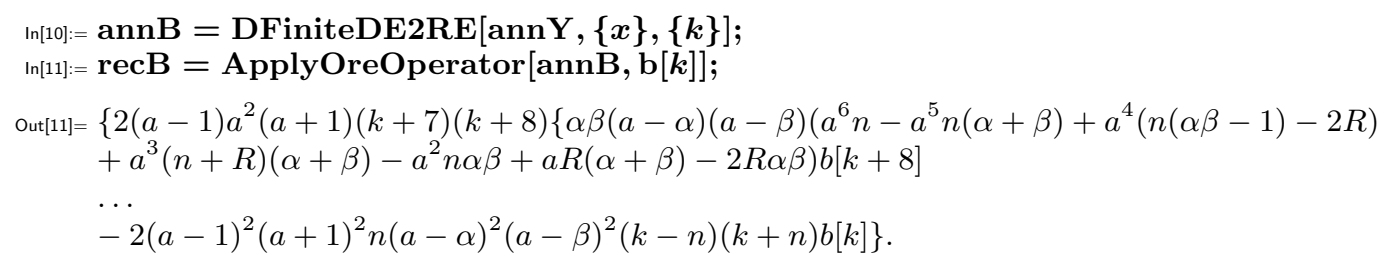

Here we omit the full output because of size and abbreviate the root expression $R=$ $\sqrt{\left(a^{2}-1\right)(a-\alpha)(a-\beta)}$ for better readability. The resulting recurrence is of order eight, because the polynomial coefficients of the given ordinary differential equation are up to degree eight. The polynomial coefficients in the recurrence are of degree two in the variable $k$ each.

The Taylor series expansion of $1 /(x-a)^{2}$ around $x_{0}=0$ is well known and thus we have

$$
y_{n}(x)=\sum_{k \geqslant 0} b_{k} x^{k}=\frac{1}{(x-a)^{2}}-p_{n}^{*}(x)=\sum_{k \geqslant 0}(k+1)\left(\frac{1}{a}\right)^{k+2} x^{k}-\sum_{k=0}^{n} c_{k} x^{k} .
$$

In other words, $b_{k}=(k+1) a^{-k-2}$ for $k>n$, which is also reflected by the factor $n-k$ in the trailing coefficient of the recurrence above. Given a recurrence for the coefficients of $y_{n}(x)$ and a recurrence for $(k+1) a^{-k-2}$ (that is easily calculated), by holonomic closure properties a recurrence for the coefficients $c_{k}$ of the polynomial of best uniform approximation can be computed:

\footnotetext{
${ }^{\dagger}$ Available at http://www.risc.jku.at/research/combinat/software/.
} 

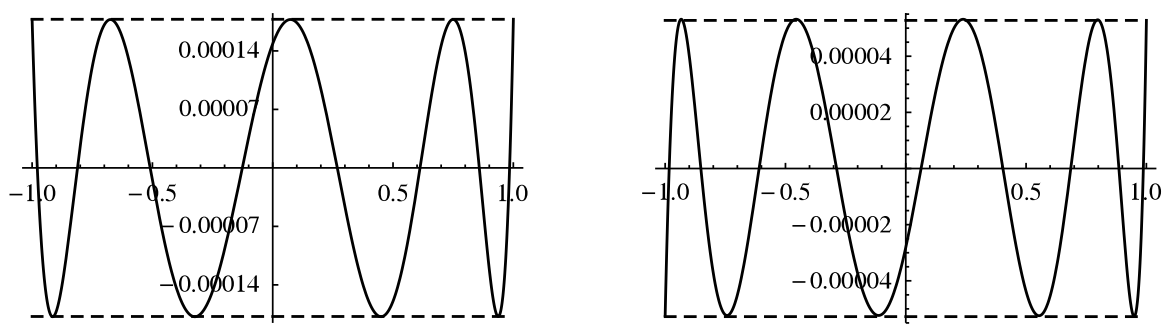

FIGURE 2. Error function for $n=7,8$ for the approximate polynomials $\tilde{p}_{n}^{*}(x)$.

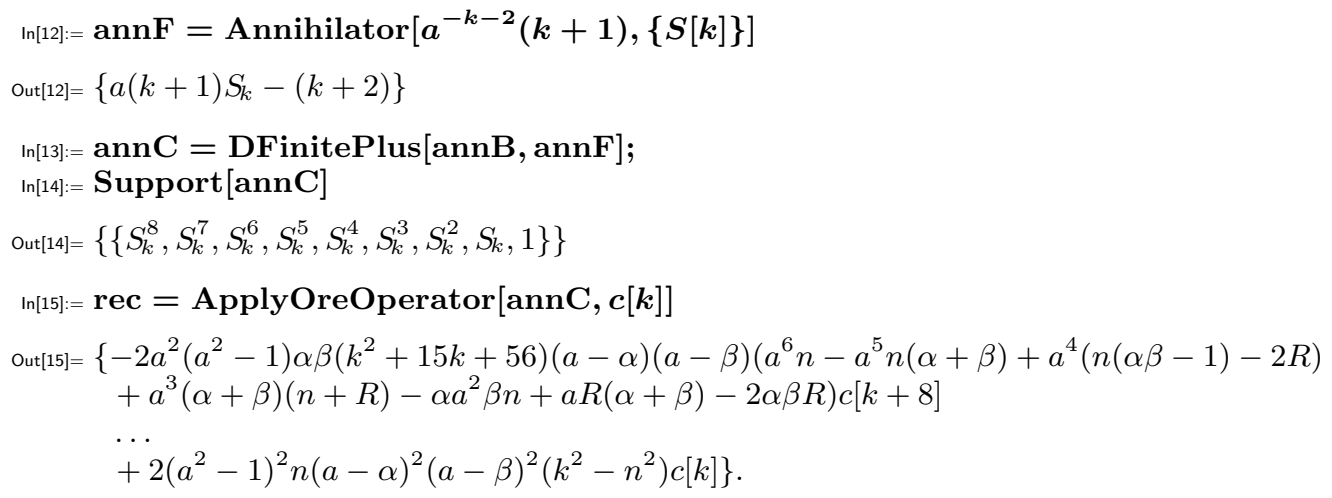

As can be seen from the Mathematica output above, the resulting recurrence is also of order eight, where we do not display the full output ${ }^{\dagger}$ and reuse the abbreviation $R=$ $\sqrt{\left(a^{2}-1\right)(a-\alpha)(a-\beta)}$. For the recursive evaluation of the coefficients we follow the approach in $[\mathbf{1 9}]$. First of all, we need the numerical values from the table of the previous section in order to initialize the parameters $\alpha$ and $\beta$. The recurrence is unwound starting from the coefficient $c_{n}$ down to $c_{0}$. As initial value first we normalize $c_{n}=1$ and, since we know that $p_{n}^{*}(x)$ is polynomial, all $c_{n+m}$ for $m=1, \ldots, 7$ are set to zero. In the second step the coefficients are adjusted to match $L_{n}$ as the minimum deviation. Using the precomputed values from the previous section, $\alpha, \beta$ and $L_{n}$ can be computed and using the recurrence relation we obtain the approximations

$$
\begin{aligned}
\tilde{p}_{7}^{*}(x)= & 0.0378802 x^{7}+0.056484 x^{6}+0.0243862 x^{5}+0.0569877 x^{4} \\
& +0.13294 x^{3}+0.192055 x^{2}+0.249238 x+0.249851
\end{aligned}
$$

and

$$
\begin{aligned}
\tilde{p}_{8}^{*}(x)= & 0.0227427 x^{8}+0.0345011 x^{7}+0.0110035 x^{6}+0.0303003 x^{5}+0.0854089 x^{4} \\
& +0.129983 x^{3}+0.186372 x^{2}+0.249608 x+0.250028
\end{aligned}
$$

for the polynomials of best uniform approximation for degree $n=7$ and $n=8$, respectively, and $a=2$. Figure 2 displays the error functions of best approximation in these cases. It is clearly visible that the approximation is a rather accurate one, showing also the required number of alternation points, at least approximately.

In [19] a recursive evaluation for the Zolotarev polynomials in Chebyshev expansion is also given. We note that such a recurrence for the coefficients of $y_{n}(x)$ can be obtained similarly

\footnotetext{
${ }^{\dagger}$ For the complete recurrence see the Mathematica notebook available for download at http://www.risc.jku. at/people/vpillwei/Results.nb.
} 
entirely automatically using the results of Benoit and Salvy [4]. Certainly it would be desirable to have a recurrence relation in $n$ for the polynomials $p_{n}^{*}(x)$. At the current stage this is beyond the capabilities of the available symbolic tools, but it remains an interesting open question.

Another possible direction for further investigations, as pointed out by one of the referees, is the application of the polynomials derived in this paper to approximating solutions of systems of linear equations $A x=b$, with $A$ symmetric but indefinite. So far in this setting the polynomials of best uniform approximation to $1 / x$ and $1 / x^{2}$ on the union of two symmetric intervals $[-\beta,-\alpha] \cup[\alpha, \beta]$ were used, which can be given in terms of Chebyshev polynomials of the first kind; see, for example, [1, p. 249] and also [17] for the case $\beta=1$, and [16], respectively.

\section{References}

1. N. I. AChIESER, Theory of approximation (Dover Publications Inc., New York, 1992). Translated from the Russian and with a preface by Charles J. Hyman, reprint of the 1956 English translation.

2. N. I. AkHIESER, Elements of the theory of elliptic functions, Translations of Mathematical Monographs 79 (American Mathematical Society, Providence, RI, 1990). Translated from the second Russian edition by H. H. McFaden.

3. G. Almkvist and D. Zeilberger, 'The method of differentiating under the integral sign', J. Symbolic Comput. 10 (1990) 571-591.

4. A. Benoit and B. SAlvy, 'Chebyshev expansions for solutions of linear differential equations', ISSAC'09: Proceedings of the Twenty-Second International Symposium on Symbolic and Algebraic Computation (ed. J. May; ACM, 2009) 23-30.

5. S. Bernstein and C. De la Vallée Poussin, L'approximation (Chelsea Publishing Co., Bronz, NY, 1970).

6. A. Bogatyrev, Extremal polynomials and Riemann surfaces, Springer Monographs in Mathematics (Springer, Heidelberg, 2012). Translated from the 2005 Russian original by Nikolai Kruzhilin.

7. M. Bronstein, Symbolic integration. I. Transcendental functions, 2nd edn, Algorithms and Computation in Mathematics 1 (Springer, Berlin, 2005), with a foreword by B. F. Caviness.

8. B. Buchberger, 'Ein Algorithmus zum Auffinden der Basiselemente des Restklassenringes nach einem nulldimensionalen Polynomideal (An algorithm for finding the basis elements in the residue class ring modulo a zero dimensional polynomial ideal)', PhD Thesis, Mathematical Institute, University of Innsbruck, Austria, 1965. English translation in J. Symbolic Comput., Special issue on logic, mathematics, and computer science: interactions. vol. 41, 475-511, 2006.

9. F. Chyzak, 'Fonctions holonomes en calcul formel', Thèse universitaire, École polytechnique, 1998. INRIA, TU 0531. 227.

10. D. Cox, J. Little and D. O'Shea, 'An introduction to computational algebraic geometry and commutative algebra', Ideals, varieties, and algorithms, 3rd edn, Undergraduate Texts in Mathematics (Springer, New York, 2007).

11. W. Decker, G. M. Greuel, G. Pfister and H. Schönemann, Singular 3-1-3 - A computer algebra system for polynomial computations. 2011. http://www.singular.uni-kl.de.

12. M. Kauers and V. Levandovskyy, 'An interface between mathematica and singular', Technical Report 2006-29, SFB F013, 2006.

13. C. Koutschan, 'Computer algebra algorithms for $\partial$-finite and holonomic functions', PhD Thesis, RISC SFB F013, J. Kepler University, 2008.

14. C. Koutschan, 'HolonomicFunctions (User's Guide)', Technical Report 10-01, RISC Report Series, University of Linz, Austria, January 2010.

15. J. Kraus, V. Pillwein and L. Zikatanov, 'Algebraic multilevel iteration methods and the best approximation to $1 / x$ in the uniform norm', Technical report, no. 2009-17, RICAM, 2009.

16. J. K. Kraus, P. S. Vassilevski and L. T. Zikatanov, 'Polynomial of best uniform approximation to $1 / x$ and smoothing in two-level methods', Comput. Methods Appl. Math. 12 (2012) 448-468.

17. I. A. Privalov, 'Approximation of $1 / x$ by polynomials on $[-1,-a] \cup[a, 1]$ ', Mat. Zametki 81 (2007) 472-473 (in Russian); translation in Math. Notes 81 (2007), 415-416.

18. M. L. Sodin and P. M. Yuditskir, 'Functions that deviate least from zero on closed subsets of the real axis. (in Russian)', Algebra i Analiz 4 (1992) 1-61; translation in St. Petersburg Math. J. 4 (1993), 201-249.

19. M. VlČEK and R. Unbehauen, 'Zolotarev polynomials and optimal FIR filters', IEEE Trans. Signal Process. 47 (1999) 717-729.

20. J. von zur Gathen and J. Gerhard, Modern computer algebra, 2nd edn (Cambridge University Press, Cambridge, 2003).

21. E. T. Whittaker and G. N. Watson, A course of modern analysis. An introduction to the general theory of infinite processes and of analytic functions: with an account of the principal transcendental functions, 4th edn (Cambridge University Press, New York, 1962). 
Ionela Moale

Research Institute for Symbolic

Computation (RISC)

Johannes Kepler University Linz

(JKU), Altenbergerstr. 69

4040 Linz, Austria

ionela.moale@risc.jku.at
Veronika Pillwein

Research Institute for Symbolic Computation (RISC)

Johannes Kepler University Linz

(JKU), Altenbergerstr. 69

4040 Linz, Austria

veronika.pillwein@risc.jku.at 